\title{
Accurate turbulence level estimations using PIV/PTV
}

\author{
Sven Scharnowski ${ }^{1} \mathbb{1} \cdot$ Matthew Bross $^{1} \cdot$ Christian J. Kähler $^{1}$
}

Received: 1 October 2018 / Revised: 6 November 2018 / Accepted: 8 November 2018 / Published online: 26 November 2018

(c) The Author(s) 2018

\begin{abstract}
The estimation of the turbulence level of flow facilities is very important for the comprehensive description of experimental results. While for low flow velocities various measurement techniques can be used (for example hot-wire, LDV, PIV) the task becomes difficult in the case of compressible flows as temperature and density fluctuations bias the measurement of the velocity fluctuations. In this work, we analyze the free-stream flow of the trisonic wind tunnel Munich (TWM) by means of particle image velocimetry (PIV) and particle tracking velocimetry (PTV). The goal is to determine the flow quality, i.e. the turbulence level, over the operating range of the facility without bias due to temperature and density variations. The capability of PIV/PTV for the estimation of small velocity fluctuations is investigated in detail. It is shown that a small particle shift on the measurement plane in combination with a large particle image displacement on the image plane allows for precise velocity measurements. Furthermore, a variation of the time separation between the PIV double images, $\Delta t$, enables the measurement uncertainty to be determined, which was estimated to be as low as $0.04 \%$ of the mean displacement for a mean displacement of $\Delta x=100$ pixel and an interrogation window size of $32 \times 32$ pixel. Regarding the wind tunnel turbulence, it was found that the turbulence level generally decreases with increasing Mach number for the TWM facility, starting with $1.9 \%$ at $M a=0.3$ and reaching $0.45 \%$ at $M a=3.0$. With this analysis, a methodology exists to perform accurate turbulence measurements in incompressible and compressible flows.
\end{abstract}

Sven Scharnowski

sven.scharnowski@unibw.de

1 Bundeswehr University Munich, Institute of Fluid Mechnics and Aerodynamics, Neubiberg, Germany 


\section{Graphical abstract}
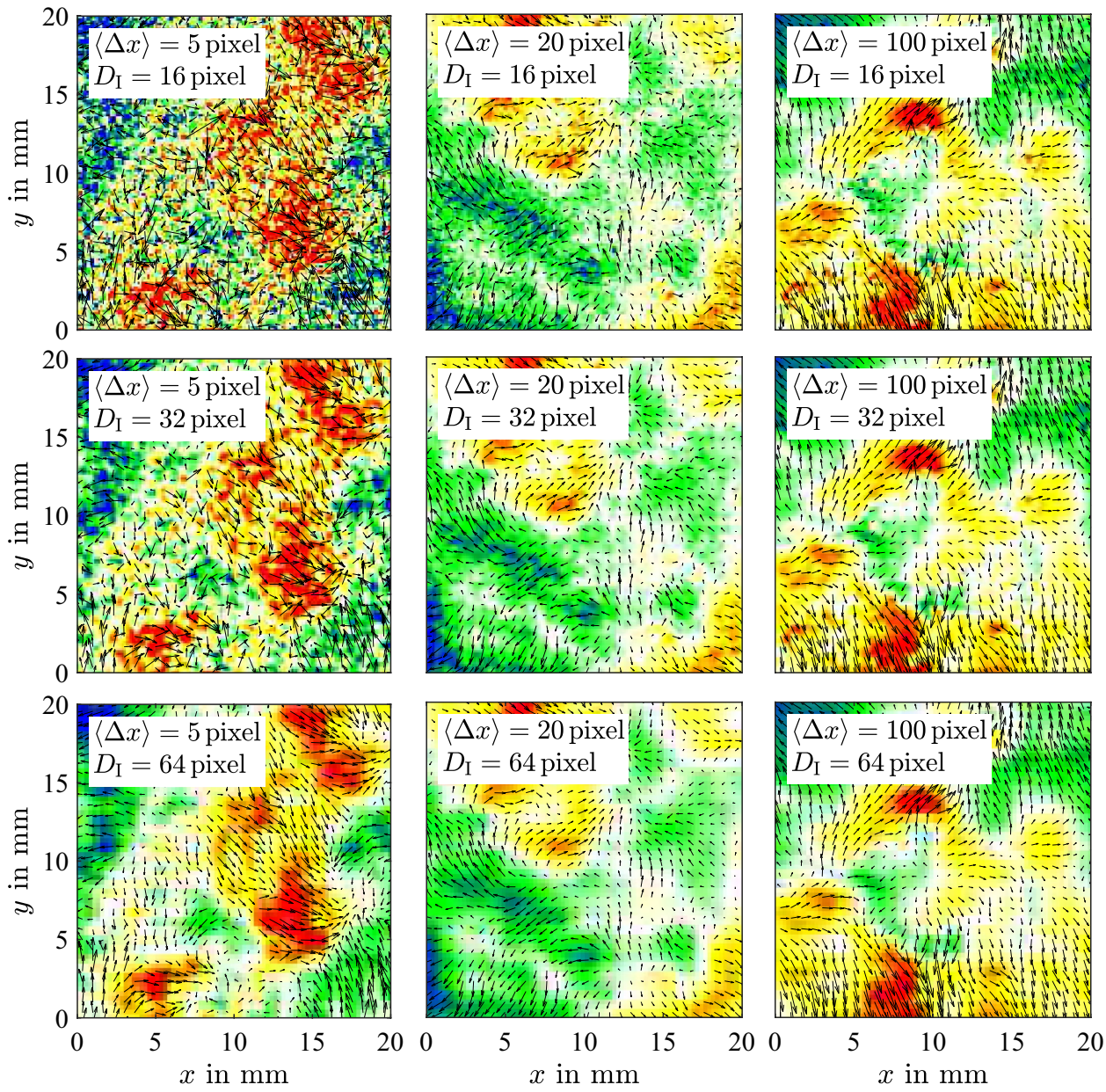

$u^{\prime}$ in $\mathrm{m} / \mathrm{s}$

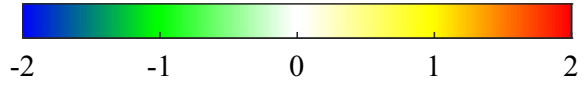

\section{Introduction}

The quantification of turbulence levels in wind tunnels is very important as many flow phenomena are very sensitive to this quantity (Ol et al. 2005). Knowing the turbulence level allows for the comparison of results measured in different facilities and to set the inflow conditions for numerical flow simulations. The turbulence level Tu is usually described by the standard deviation of the fluctuations of the streamwise velocity component normalized by the mean streamwise velocity

$\mathrm{Tu}=\sqrt{\left\langle u^{\prime 2}\right\rangle} /\langle u\rangle$

where the standard deviation is computed from the ensemble of velocity measurements $\sqrt{\left\langle{u^{\prime 2}}^{2}\right\rangle}=\sqrt{\frac{1}{N-1} \sum_{n}\left(u_{n}-\langle u\rangle\right)^{2}}$

with $\langle u\rangle$ being the mean velocity of an ensemble of independent and uncorrelated measurements.

Accurate turbulence measurements require a measurement system that has an uncertainty that is well below the velocity fluctuations of interest. Additionally, the measurement volume that each velocity vector is estimated from must be small compared to the size of the smallest turbulent structures. Furthermore, the number of samples $N$ must be sufficiently large to ensure that the average velocity is fully converged. Hot-wire probes are well suited for 
precise velocity measurements with high temporal resolution (Alfredsson et al. 1988; Hutchins et al. 2009; Hultmark et al. 2013). However, hot-wires have several drawbacks. To mention a few, they are intrusive measurement methods, results can be biased if other velocity components also have large fluctuations, temperature fluctuations influence the results, and extracting the velocity from the measured signal requires knowledge about the flow density. Furthermore, at supersonic Mach numbers, the shock waves generated by the probe will influence the measurement. Therefore, the application of hot-wire probes is usually limited to incompressible flow. A non-intrusive alternative measurement technique is the Laser-Doppler velocimetry (LDV) (George and Lumley 1973; Shirai et al. 2006). Due to its working principle LDV does not require knowledge about the flow density and is, therefore, suited for measurements in compressible flows even in the case of temperature fluctuations. Hot-wire probe and LDV are measurement techniques with the capability of high temporal resolution, however, they only provide point-wise information about the flow. To analyze the spatial organization of turbulent structures planar or volumetric measurement techniques are needed. Particle image velocimetry (PIV) provides such spatial information which corresponds up to $\mathrm{MHz}$ sampling locally taking the convection velocity into account. However, PIV is known to have higher measurement uncertainties than hot-wire probes and LDV, in general (Wilson and Smith 2013; Neal et al. 2015; Timmins et al. 2012). Although systematic errors would not affect the estimation of Tu, according to Eq. (1), it is important to quantify random errors, since they increase the estimated velocity fluctuations. This is discussed in detail in Sect. 3.5.

It was already shown in the past that time-resolved sequences of PIV and particle tracking velocimetry (PTV) recordings allow for improved measurement uncertainty (Hain and Kähler 2007; Schröder et al. 2015; Cierpka et al. 2013; Sciacchitano et al. 2012; Willert et al. 2018; Scarano and Moore 2012). However, time-resolved measurements are limited to flow velocities around $10 \mathrm{~m} / \mathrm{s}$, for a recording rate in the low $\mathrm{kHz}$ range. In order to achieve several 100 's of $\mathrm{kHz}$, as needed for a detailed analysis of supersonic flows, the number of images and/or the image size is usually rather limited (Wernet and Opalski 2004; Beresh et al. 2017). Furthermore, reliably estimating flow statistics from time-resolved measurements requires very long sequences leading to an amount of data that is challenging to handle and to evaluate.

The aim of this work is to investigate the capability of double-pulse PIV/PTV to estimate the wind tunnel turbulence level for free-stream velocities between $u_{\infty}=100 \mathrm{~m} / \mathrm{s}$ and $600 \mathrm{~m} / \mathrm{s}$. Since velocity fluctuations on the order of $1 \%$ are expected, the measurement uncertainty should be well below that value. In the case of PIV/PTV, the measured displacement fluctuations $\Delta x^{\prime}=\Delta x-\langle\Delta x\rangle$ are a combination of the actual velocity fluctuations $u^{\prime}=u-\langle u\rangle$ and the random error of the displacement measurement, characterized by its standard deviation $\sigma_{\Delta \mathrm{x}}$. For small amounts of loss-of-correlation due to out-of-plane motion and for small gradients, the measurement uncertainty is independent of the shift vector length. Thus, both add up to the measured fluctuations as follows:

$$
\begin{aligned}
\left\langle\Delta x^{\prime 2}\right\rangle_{\text {measured }} & =\left\langle\Delta u^{\prime 2}\right\rangle \cdot \frac{\Delta t^{2}}{M^{2}}+\sigma_{\Delta \mathrm{x}}^{2} \\
& =\left\langle\Delta x^{\prime 2}\right\rangle_{\text {turb }}+\sigma_{\Delta \mathrm{x}}^{2}
\end{aligned}
$$

where $M$ is the optical magnification and $\Delta t$ stands for the time separation between the double images. To ensure highly accurate measurements with PIV/PTV $\Delta t$ must be optimized to maximize the dynamic velocity range (Adrian 1997). On the one hand, the particle image displacement on the camera sensor must be large to achieve low relative measurement uncertainty (Scharnowski and Kähler 2016a). On the other hand, the particle displacement on the physical plane must be small to avoid loss-of-pairs due to out-of-plane motion as well as filtering effects due to gradients (Scharnowski and Kähler 2013; Scharnowski et al. 2017).

To perform reliable turbulence level estimations by means of PIV/PTV, the measurement setup and the evaluation procedure must be selected carefully to minimize the measurement uncertainty. In the following the test facility, the measurement setup and the data evaluation are described in detail. Further on, the effect of the particle image displacement and the interrogation window size on the estimated turbulence level is evaluated. Finally, conclusions are drawn from the presented results.

\section{Measurement setup}

The measurements were performed in the trisonic wind tunnel at the Bundeswehr University in Munich (TWM). The TWM facility is a blow-down type wind tunnel with a 300 $\mathrm{mm}$ wide and $675 \mathrm{~mm}$ high test section, as shown in Fig. 1. The facility is discussed in detail in Bolgar et al. (2018). Two adjustable throats, the Laval nozzle upstream of the test section and the diffuser further downstream, enable an operating range of Mach numbers from 0.2 to 3.0. The facility has two tanks that can be pressurized up to 20 bar above ambient pressure, holding a total volume of $356 \mathrm{~m}^{3}$ of dry air. To control the Reynolds number, the total pressure in the test section is varied between 1.2 and 5 bar. The wind tunnel working range with respect to Reynolds number and Mach number is illustrated in Fig. 2. The smallest possible cross section of the diffuser defines the lowest Mach number and 


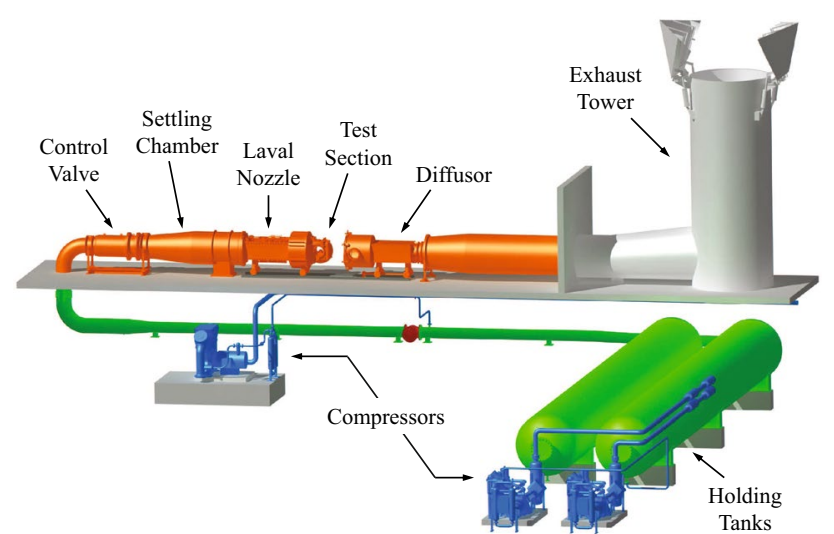

Fig. 1 Sketch of the trisonic wind tunnel Munich

the smallest cross section of the Laval nozzle defines the highest Mach number. The upper Reynolds-number limit is determined by the maximum total pressure of $p_{0}=5 \mathrm{bar}$ and the lower Reynolds number is limited by $p_{0}=1.2 \mathrm{bar}$ for subsonic flow and an increasing pressure, that allows for adaptive nozzle conditions, for supersonic flow. To characterize the wind tunnel, PIV measurements were performed for 9 different Mach numbers ranging from $M a=0.3$ to 3.0.

For the PIV measurements, the flow was seeded with Di-Ethyl-Hexyl-Sebacat (DEHS) tracer particles with a mean diameter of $1 \mu \mathrm{m}$, as described by Kähler et al. (2002). The size of the tracers is a compromise between visibility and the ability to follow the flow. The response time of these droplets is about $2 \mu$ s (Ragni et al. 2011),

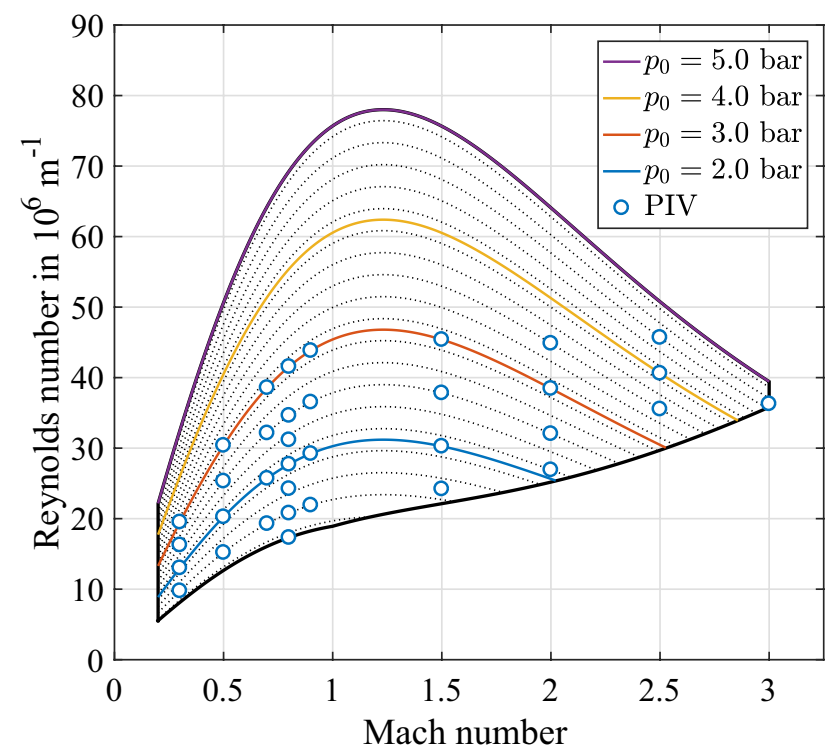

Fig. 2 TWM working range for Mach number and Reynolds number as a function of the total pressure $p_{0}$ and PIV measurement points which is considered to be sufficient for investigations at the selected Mach and Reynolds numbers since no strong turbulent fluctuations are to be expected in the free-stream. The tracers were illuminated by a $500 \mu \mathrm{m}$ thick light sheet in a horizontal plane at the center of the test section. The scattered light of the tracer particles was imaged onto the sensor of a sCMOS camera with double image capability and global shutter by means of a $100 \mathrm{~mm}$ lens in combined with a $2 \times$ tele-converter. The f-number of the lens aperture was set to 8 in order to reduce aberrations and to ensure sufficiently large particle images. Double images were recorded at a frequency of $15 \mathrm{~Hz}$. The resulting size of the field of view is $26 \mathrm{~mm} \times 22 \mathrm{~mm}$ and the resulting scaling factor is $10.4 \mu \mathrm{m} /$ pixel. With this PIV setup, a mean particle image diameter of about 3.4 pixel was achieved. From the auto-correlation function of the images a background noise level with a standard deviation of about 50 counts was estimated with the method presented in Scharnowski and Kähler (2016b). This noise level leads to a loss-ofcorrelation due to image noise of $F_{\sigma} \approx 0.91$ and to a signalto-noise ratio of $\mathrm{SNR} \approx 3.2$, which is considered to be well suited for accurate PIV evaluation, according to Scharnowski and Kähler (2016b). Figure 3 shows a small section of a typical PIV image pair and the corresponding correlation function. It can be seen from the image that most particle images can be paired although the mean displacement was set to $\langle\Delta x\rangle=100$ pixel. Consequently, a correlation function with a well detectable peak was computed.
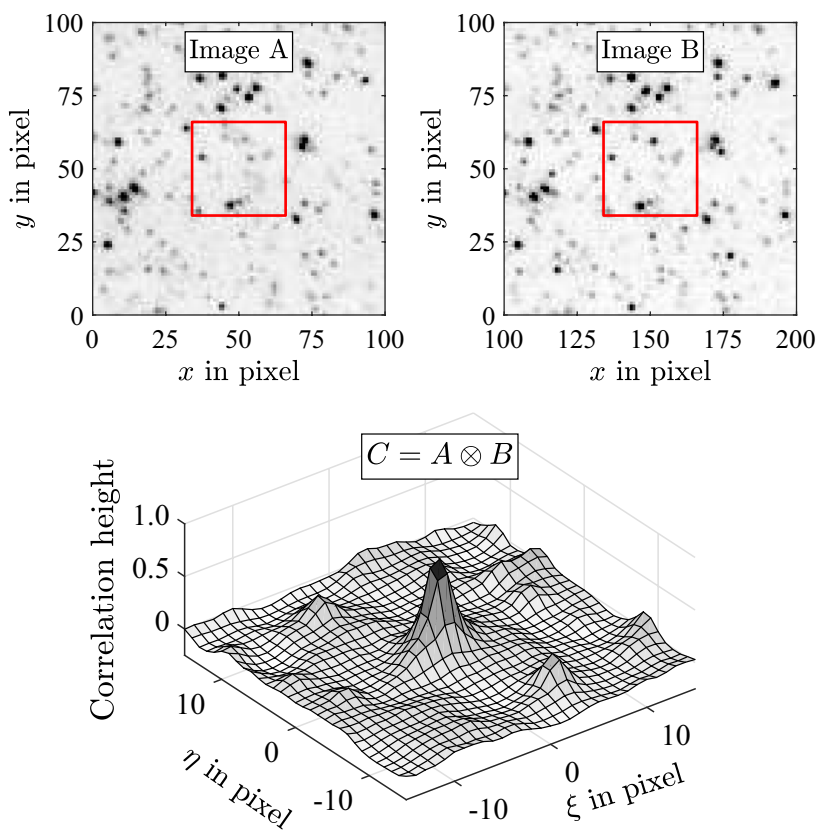

Fig. 3 Section of a typical PIV image pair (top) and correlation peak for the marked region (bottom). The section of image B is shifted by the mean displacement $\langle\Delta x\rangle=100$ pixel 


\section{Results and discussions}

\subsection{Wind tunnel stability}

The run time of the TWM facility is limited by the mass of pressurized air in the holding tanks. Depending on the mass flow, which is a function of the Mach number and the Reynolds number, the usable run time is between $50 \mathrm{~s}$ and $3 \mathrm{~min}$ for the analyzed configurations, refer to Fig. 2. At the beginning of a wind tunnel run the control valve (see. Fig. 1) opens quickly until the desired total pressure is reached and thereafter opens contentiously to compensate for the decreasing pressure in the holding tanks. The control valve's motion is controlled in a closed loop to hold the total pressure constant, which is measured in the settling chamber. Figure 4 illustrates the resulting pressure fluctuations in the test section of the facility. The figure clearly shows that high fluctuations occur in the beginning of each run where the reservoir pressure is close to 20 bar. Thereafter, the fluctuations decrease and stay at a rather constant level of about 1 to $1.3 \%$ until the reservoir pressure drops to a pressure of 5 bar. At the end of the wind tunnel run the pressure fluctuation are the lowest because the control valve is open fully and most sensitive. The duration of rather constant pressure fluctuations is the usable measurement time during which the presented data was acquired.

\subsection{Effect of number of recordings}

Estimating statistics of turbulent flows by means of PIV measurement with a relatively slow acquisition rate requires

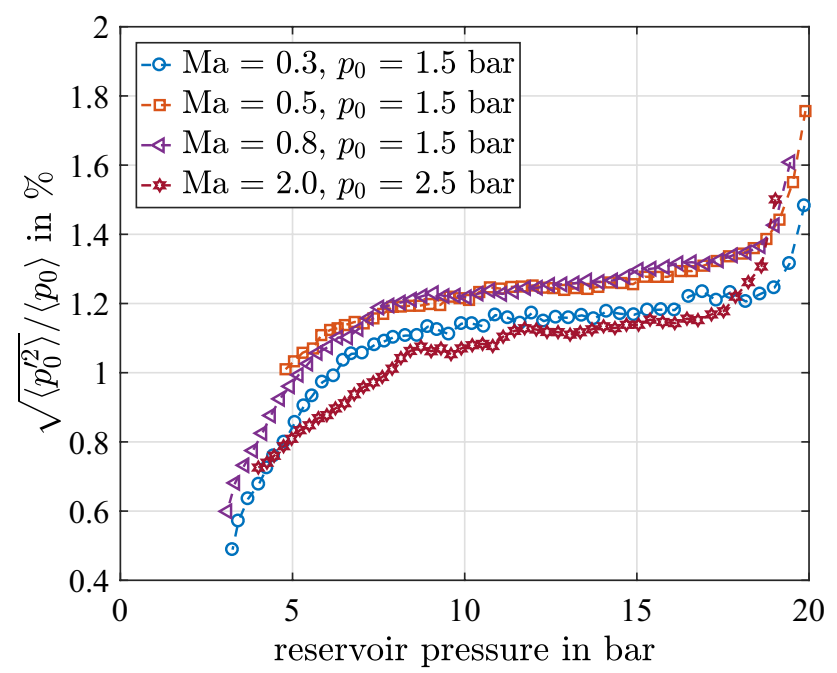

Fig. 4 Pressure fluctuations in the test section of the trisonic wind tunnel as a function of the reservoir pressure for different Mach numbers a certain number of recordings (Kähler et al. 2006, 2016). Figure 5 shows how the turbulence level estimation depends on the number of PIV recordings evaluated. The markers in the figure represent the spatially averaged turbulence level within the field of view with respect to the number of PIV recordings. The error bars correspond to the standard deviation of the spatial variation of the estimated turbulence level. It is evident from Fig. 5 that the spatial variation decreases with increasing number of recorded images, as expected. Furthermore, the estimated turbulence level changes with increasing number of recordings, especially for $M a=0.3$. For 100 vector fields or more the value is reasonably converged for the present experiment. To ensure reliable results, 1000 PIV double images were recorded for the $\Delta t$ variation in the following section. For the variation of the Reynolds number and the Mach number, 500 recordings were measured for all datasets.

\subsection{Effect of $\Delta t$ and window size}

To analyze the effect of the time between the double images $\Delta t$ and the squared interrogation window size $D_{\mathrm{I}}$ on the estimated turbulence level, these parameters were varied for a Mach number of $M a=0.3$ and a total pressure of $p_{0}=1.5$ bar. Based on the findings of Sect. 3.2, 1000 double images were recorded for nine different $\Delta t$ between $0.2 \mu \mathrm{s}$ and $20 \mu \mathrm{s}$, corresponding to a mean particle image shift between $\Delta x \approx 2$ pixel and 200 pixel. The PIV recordings were evaluated using state-of-the-art PIV software from LaVision $\mathrm{GmbH}$ (DaVis 8.3) including multi-pass image deformation and Gaussian window weighting. The final window size was varied between $12 \times 12$ pixel and $64 \times 64$ pixel.

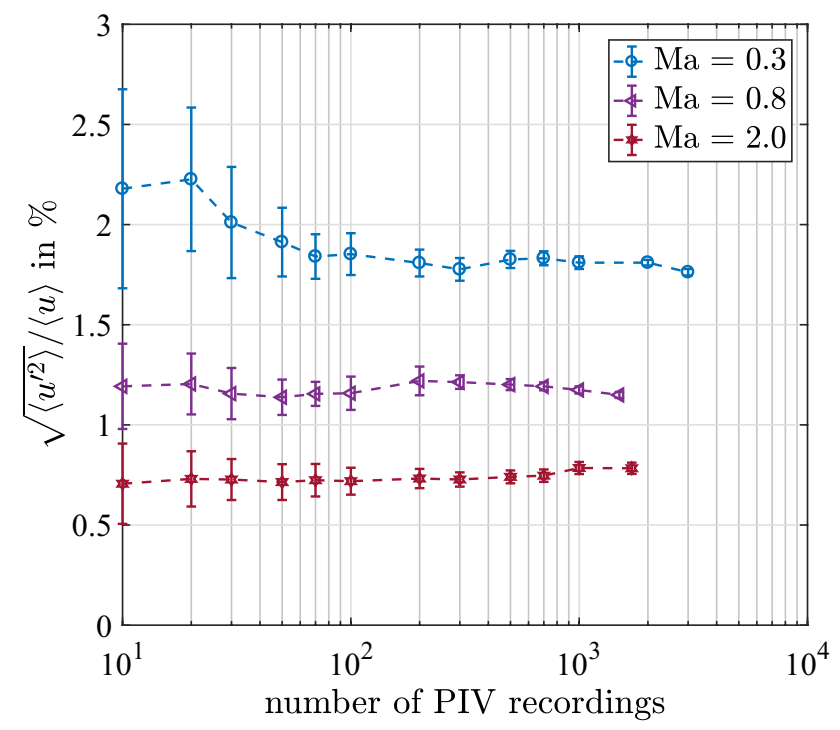

Fig. 5 Estimated turbulence intensity as a function of the number of PIV velocity fields acquired at $15 \mathrm{~Hz}$ for $M a=0.3$ and $p_{0}=1.5 \mathrm{bar}$ 
For a window size of 12 or 16 pixel no window overlap was applied, while for larger $D_{\mathrm{I}}$ a window overlap of $50 \%$ was used.

The results for the variation of the particle image shift $\Delta x$ and different interrogation window sizes $D_{\mathrm{I}}$ are illustrated in Fig. 6 by means of example velocity fields. For each column in the figure the same PIV recordings were evaluated. It can be seen from the top left image in the figure, that with decreasing displacements and decreasing interrogation window size the noise is amplified. It is obvious that under these conditions the measurement uncertainty is too high to reliably estimate the velocity fluctuations. For increasing $\Delta x$ as well as for increasing $D_{\mathrm{I}}$ the vector fields appear much smoother and are, therefore, better suited for turbulence level estimations. The fields are less noisy because the uncertainty of the estimated velocity is reduced by the decreased absolute uncertainty of the particle image displacement $\sigma_{\Delta \mathrm{x}}$ (in the case of larger $D_{\mathrm{I}}$ ) or by decreased relative shift vector uncertainty due to enlarged mean displacement $\langle\Delta x\rangle$. However, care must be taken when increasing $\Delta x$ or $D_{\mathrm{I}}$ for two reasons. First, the larger the particle image displacement becomes the higher is the risk of introducing bias errors due to curved streamlines, which are not captured in double-pulse PIV experiments (Scharnowski and Kähler 2013). These bias errors would also affect the Tu estimation, since they vary within the flow field. Second, larger interrogation windows cause increased spatial low-pass filtering of the velocity field, leading to smoothed results which might filter out small-scale turbulent structures (Scharnowski et al. 2012). To minimize these two effects, a small field of view was selected such that the interrogation window sizes as well as the particle image displacement are small when projected on the measurement plane.

How the combination of the different $\Delta t$ is used to determine the turbulence level is discussed in detail in Sect. 3.5, together with a discussion on the effect of spatial filtering due to the window size.
Fig. 6 Example velocity fields for a Mach number of $M a=0.3$ and a total pressure of $p_{0}=1.5$ bar computed from PIV recordings with different mean particle image displacements $\langle\Delta x\rangle$ (increasing from left to right) and different interrogation window sizes $D_{\text {I }}$ (increasing from top to bottom)
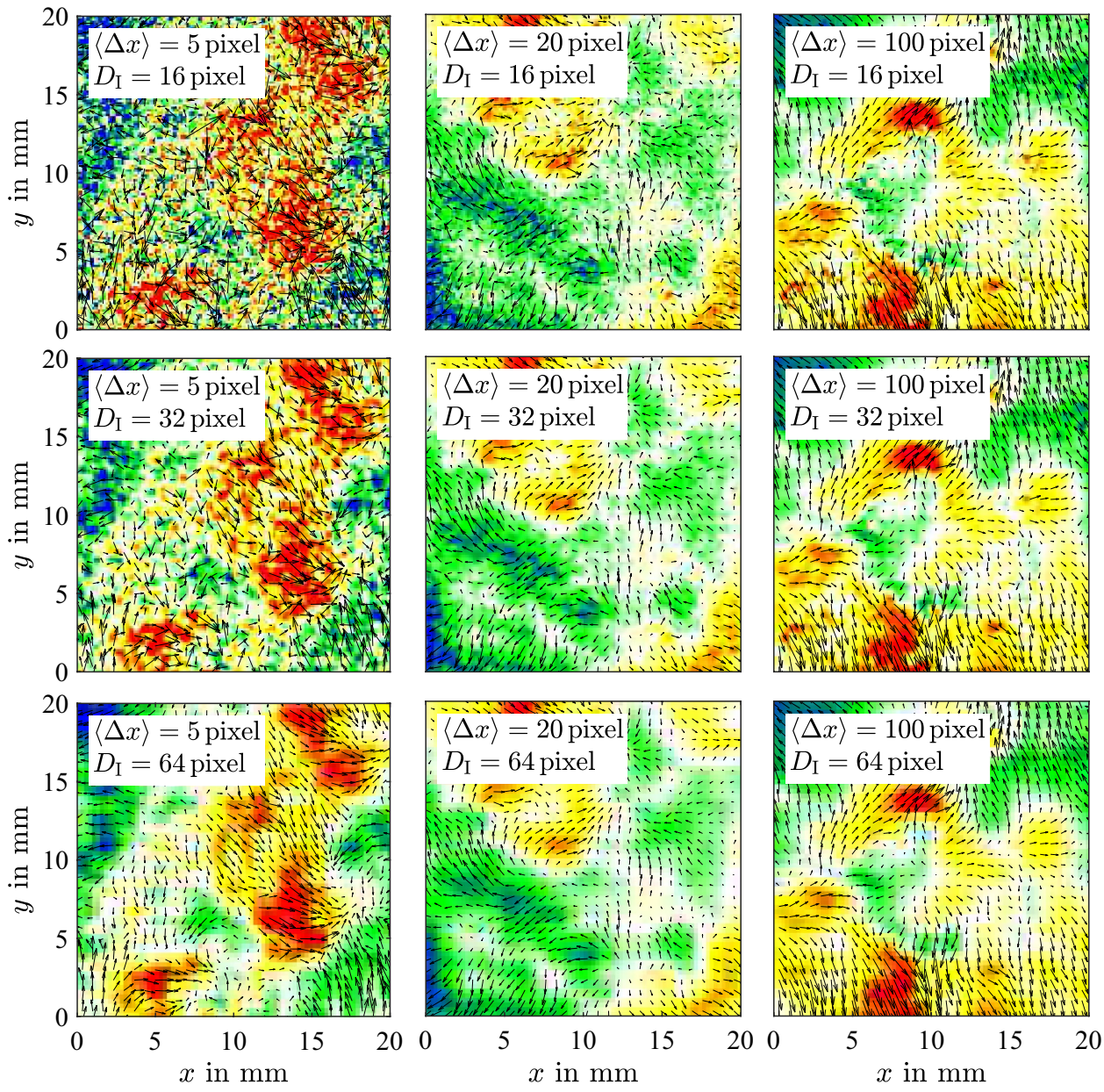

$u^{\prime}$ in $\mathrm{m} / \mathrm{s}$ 


\subsection{Peak locking}

One important error source that must be considered for PIV and PTV measurements is the so-called peak-locking effect (Raffel et al. 2018). Peak locking is a bias error that shifts the estimated displacement values towards the closest integer pixel positions and is caused by very small particle images. In the case of peak locking, the probability density function of the displacement shows peaks at integer pixel values (Kähler 1997; Christensen 2004; Overmars et al. 2010; Raffel et al. 2018). As a result, the velocity is overestimated or underestimated depending on the sub-pixel length of the shift vector on the image plane.

To evaluate the significance of the peak-locking effect for a specific measurement, it was demonstrated in Nerger et al. (2003) that velocity histograms for measurements with different $\Delta t$ differ strongly in the case of peak locking. Only if peak locking is negligible the velocity histograms appear similar as only the random error varies. For reliable velocity measurements it is important to avoid peak locking during the data acquisition using appropriate optical setups (Michaelis et al. 2016; Kislaya and Sciacchitano 2018), short observation distances or cameras with small pixel size.

For the here presented PIV images, peak locking was prevented by using a high-quality lens in combination with a short working distance and a camera sensor with a small pixel pitch. To prove that the data is not biased by peak locking, Fig. 7 shows the histogram of the sub-pixel streamwise displacement computed from 1000 velocity fields with a mean displacement of $\langle\Delta x\rangle \approx 100$ pixel and an interrogation window size of $32 \times 32$ pixel. With peak locking, the histogram would show higher probability for displacements close to 0 or 1 pixel. Instead the histogram in Fig. 7 has a flat distribution, proving the absence of peak locking.

\subsection{Turbulence level estimation}

The turbulence level combines all possible velocity fluctuations in a single value. For a Gaussian distribution of the

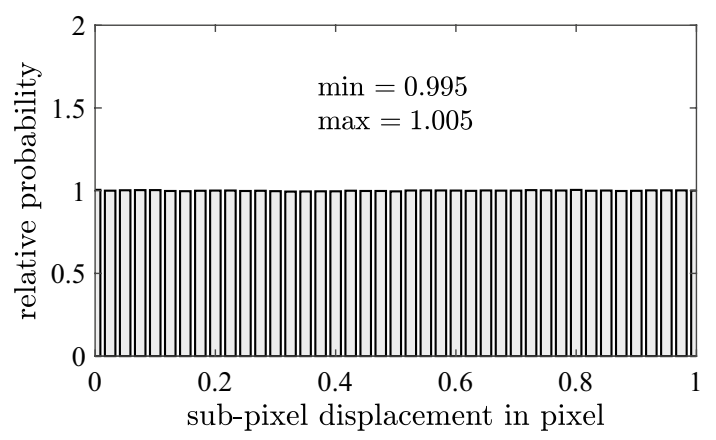

Fig. 7 Histogram of the streamwise sub-pixel displacement for 1000 velocity fields at $M a=0.3$ and $p_{0}=1.5 \mathrm{bar}$ velocity fluctuations the standard deviation is sufficient to fully describe the probability of all velocities. To verify that the distributions are indeed Gaussian, the histogram of the velocity fluctuations is illustrated in Fig. 8 for different mean shift vector lengths $\langle\Delta x\rangle$. The figure shows that all tested particle image displacements result in smooth histograms without multiple peaks. This confirms again the absence of peak locking. The differences in the distribution widths are caused by the measurement uncertainty according to Eq. (3).

Since the different $\Delta x$ result in different distributions of the velocity fluctuations, the question arises which shift is suitable for the determination of the turbulence level. Unfortunately, there is a risk that none of the individual $\Delta x$ values is suitable. This is because the measurement uncertainty is unknown in general. To overcome this issue, all the different data sets can be combined to extract the unknown measurement uncertainty. Dividing Eq. (3) by the mean displacement squared $\langle\Delta x\rangle^{2}$ results in the following relation:

$\frac{\left\langle\Delta x^{\prime 2}\right\rangle_{\text {measured }}}{\langle\Delta x\rangle^{2}}=T u^{2}+\frac{\sigma_{\Delta x}^{2}}{\langle\Delta x\rangle^{2}}$

where $\left\langle\Delta x^{\prime 2}\right\rangle_{\text {measured }}$ and $\langle\Delta x\rangle$ are known but Tu and $\sigma_{\Delta \mathrm{x}}$ are unknown.

Since several value pairs are available for the mean shift vector and the measured fluctuations, the unknown Tu and $\sigma_{\Delta \mathrm{x}}$ can be estimated from a fit function, according to Eq. (4). The results for the different $\Delta x$ and $D_{\mathrm{I}}$ are shown in Fig. 9. For comparison PTV results are also shown.

The symbols in the figure represent the spatial mean of the temporal standard deviation and the error bars correspond to the spatial variations within the field of view. The dashed lines indicate the distribution of a fit-function using

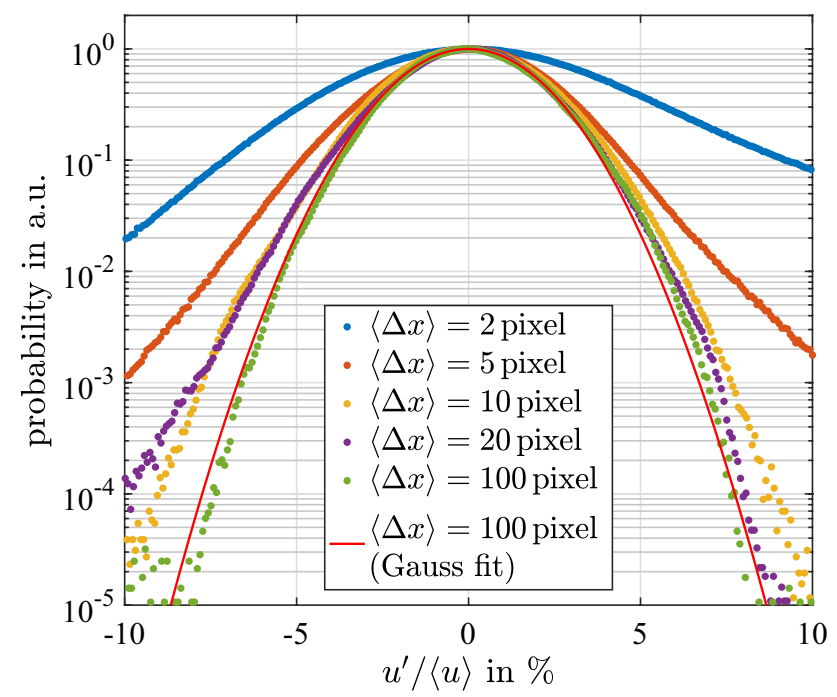

Fig. 8 Histogram of the estimated velocity fluctuations for different particle image displacements $\Delta x$ at $M a=0.3$ and $p_{0}=1.5 \mathrm{bar}$ 


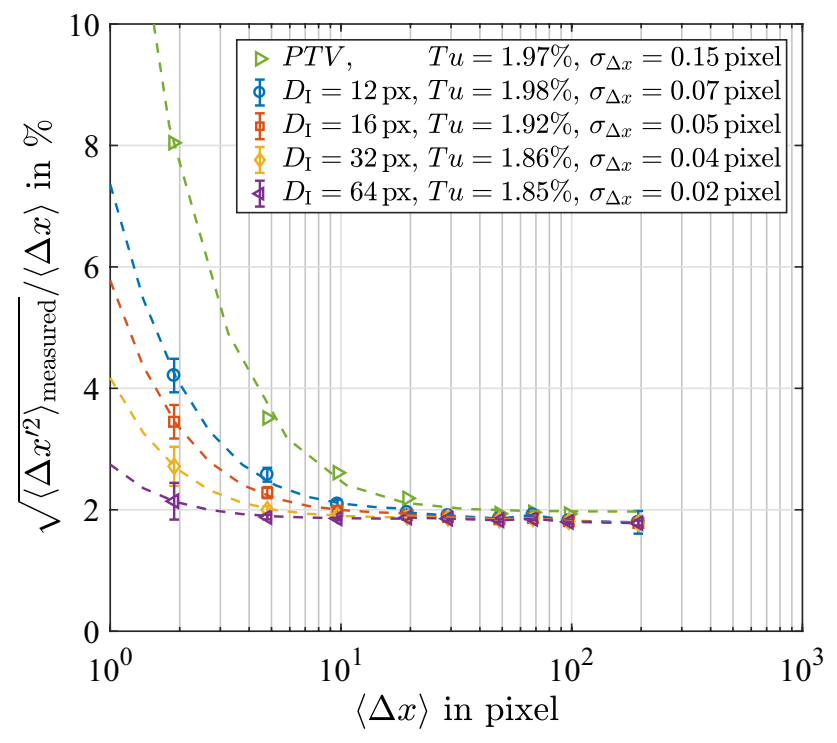

Fig. 9 Estimated turbulence intensity as a function of the particle image displacement $\Delta x$ for different window sizes at $M a=0.3$ and $p_{0}=1.5 \mathrm{bar}$

Eq. (4). Values for the fit-parameters Tu and $\sigma_{\Delta \mathrm{x}}$ are given in the figure. As expected, the estimated turbulence level increases with decreasing interrogation window size indicating that some of the turbulent structures are smaller than the interrogation windows. However, since the changes in the $\mathrm{Tu}$ values are very small it can be concluded that most structures are larger than 64 pixel $(=670 \mu \mathrm{m})$. Furthermore, the PTV results, which do not suffer from spatial low-pass filtering, confirm the PIV results of the smallest tested interrogation windows.

For the particle tracking velocimetry (PTV) evaluation, the 2D locations of the particle images was identified by applying 2D Gaussian fitting functions to the particle images, rejecting particle images that exceed a defined intensity threshold or min/max pixel size. The tracking of corresponding particle image pairs was performed by a non-iterative double frame particle tracking approach (Fuchs et al. 2017). Due to the high seeding concentration which was optimized for PIV, overlapping particle images appear frequently. This has a strong effect on the measurement uncertainty (Kähler et al. 2012). Furthermore, it has to be taken into account that PIV requires just one peak center estimation while PTV requires two, which raises the uncertainty in this PTV evaluation.

Besides the turbulence level, the fitting parameters in Fig. 9 also provide the measurement uncertainty $\sigma_{\Delta x}$. As expected the measurement uncertainty increases with decreasing window size. For the PTV evaluation $\sigma_{\Delta \mathrm{x}}$ is slightly larger than for PIV, due to the aforementioned reasons.
In the last years several approaches for determine the uncertainty of PIV measurements were developed (Xue et al. 2015; Wieneke 2015; Timmins et al. 2012; Sciacchitano et al. 2013, 2015; Sciacchitano and Wieneke 2016; Neal et al. 2015; Wilson and Smith 2013; Charonko and Vlachos 2013; Christensen and Scarano 2015). If the uncertainty of the PIV velocity fields is known a variation of $\Delta t$ is not necessary for the estimation of $\mathrm{Tu}$, in principle. However, the different approaches seem to have varying sensitivities and result in different uncertainty bounds. A variation of $\Delta t$ is more complex than a single measurement but the present analysis indicates that this is a reliable way.

\subsection{Wind tunnel characterization}

Based on the presented sensitivity analysis, the parameters for a reliable estimation of the wind tunnel turbulence level over the full range of possible Mach numbers and Reynolds numbers were selected. To minimize the wind tunnel run time, only one run with 500 densely seeded image pairs and a particle image shift of $\langle\Delta x\rangle \approx 100$ pixel was performed for each wind tunnel condition. According to the findings in Fig. 9, a direct estimation of the turbulence level from only one $\Delta x$ is possible for such a large mean particle image shift. The PIV images were evaluated with a final interrogation window size of $32 \times 32$ pixel. Due to the slightly higher measurement uncertainty of PTV at densely seeded flows it was decided to use PIV for further evaluation. Alternatively, lower seeded images could be evaluated with PTV but to reach the same statistical convergence of the higher seeded flow more wind tunnel blow downs (and refills) would be needed.

The resulting turbulence levels are shown in Fig. 10. It can be clearly seen from the figure that the total pressure $p_{0}$ and thus the Reynolds number have only a minor effect on the velocity fluctuations. However, the Mach number clearly influences the turbulence level. With the exception of $M a=0.9$ the turbulence level decreases monotonically with increasing Mach number and reaches a value of $T u=0.45 \%$ for $M a=3.0$. These results are in agreement with the findings in Scharnowski et al. (2017a), where a turbulence level of $T u<1.5 \%$ at $M a=0.8$ was reported from PIV measurements using only one $\Delta t$ and a significantly coarser spatial resolution.

Besides the fluctuations of the streamwise velocity component $u$, the spanwise $v$ and the vertical $w$ components were computed. While $v$ could be analyzed from the same field of view, $w$ was measured in an additional streamwise-vertical plane. Figure 11 summarizes the turbulence intensities for the different velocity components. It can be seen that the fluctuations of the spanwise and the vertical velocity component are slightly larger than for the streamwise one. 


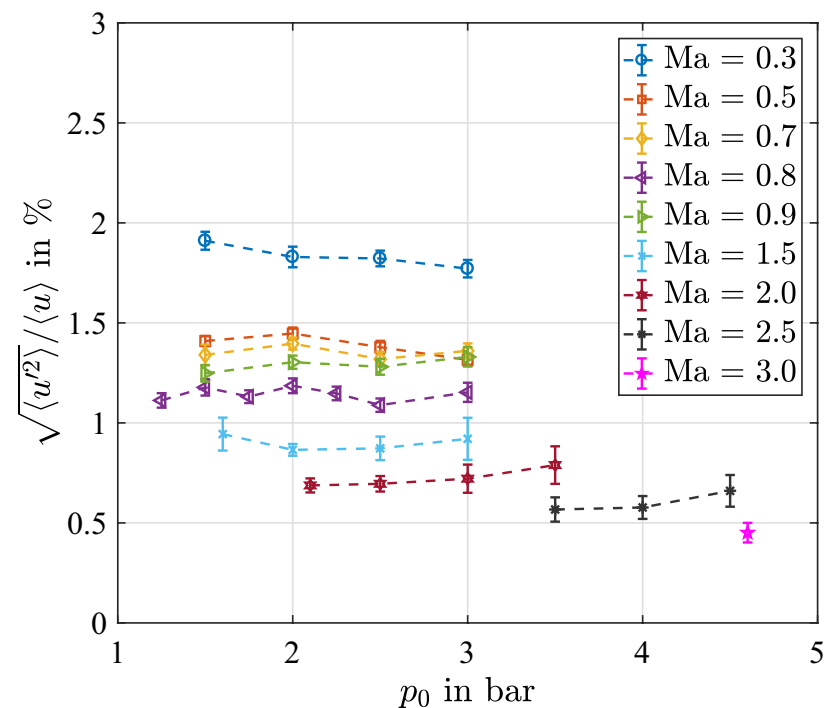

Fig. 10 Fluctuations of the streamwise velocity component $u$ with respect to the stagnation pressure $p_{0}$ for different Mach numbers

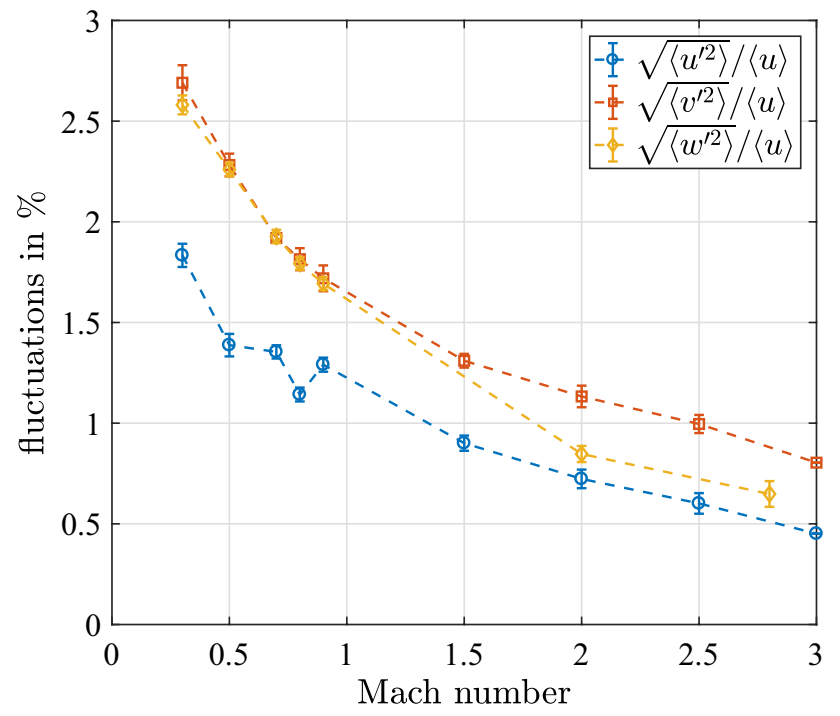

Fig. 11 Fluctuations of the three velocity components $(u, v, w)$, the static pressure $p_{\infty}$ and the stagnation pressure $p_{0}$ as a function of the Mach numbers. The error bars indicate the variation of the quantities for the different tested stagnation pressures

\subsection{Spectral analysis}

The velocity fields measured with PIV suffer from poor temporal resolution, since the acquisition rate was only $15 \mathrm{~Hz}$, however, the small field of view and the relatively large camera sensor results in a high spatial resolution. The spatial information of the PIV results can be used to perform spectral analysis in time by applying Taylor's hypothesis of frozen turbulence (Taylor 1938). This is done by multiplying the spatial frequencies with the mean velocity.

Figure 12 shows the power spectral density PSD pre-multiplied with the frequency as a function of the frequency for a Mach number of 0.3 and a variation of the mean particle image shift $\langle\Delta x\rangle$. The PSD was computed from vector fields based on an interrogation window size of $D_{\mathrm{I}}=64$ pixel with $50 \%$ overlap using the method of Welch (1967) and averaging the spectra of each streamwise line from 500 velocity fields. It is important to note that only the high-frequency end could be extracted from the PIV results since the field of view is relatively small. The highest resolved frequency is the mean velocity divided by two times the interrogation window size which is $75 \mathrm{kHz}$. The PSD can be computed for frequencies up to $150 \mathrm{kHz}$ because the velocity data is oversampled due to the window overlap. However, it is important to note that the PSD for frequencies higher than the mean velocity divided by $2 D_{\mathrm{I}}$ is within the noise floor. Thus, the resolved frequencies range from 5 to $75 \mathrm{kHz}$, which corresponds to a wavelength range of $1.3 \mathrm{~mm}$ to $20 \mathrm{~mm}$. The variation of $\langle\Delta x\rangle$ in Fig. 12 illustrates how the measurement noise contributes to the spectrum. It can be concluded that the random error mainly acts on high frequencies, as expected from the vector fields in Fig. 6.

The variation of the Mach number in Fig. 13 shows a similar trend for the subsonic Mach numbers, but the frequencies are shifted to higher values since the mean velocity increases. For the supersonic cases, a local peak seems to develop at high frequencies with increasing Mach number. This peak might be due to small-scale turbulent structures or due to increasing measurement uncertainty.

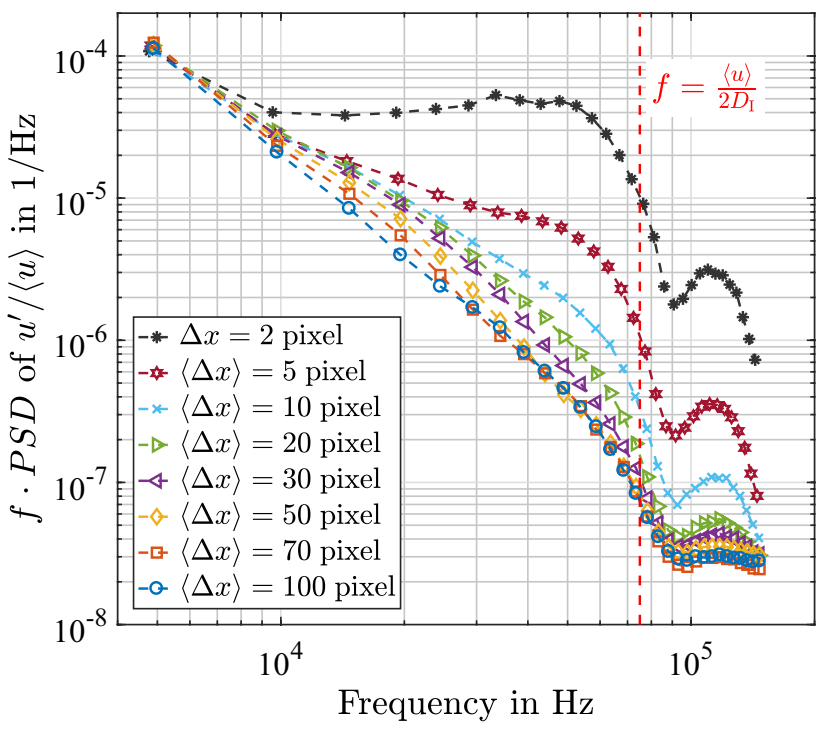

Fig. 12 Power spectral density of the normalized velocity fluctuations for different particle mean image displacements $\langle\Delta x\rangle$ at $M a=0.3$ and $p_{0}=1.5 \mathrm{bar}$ 


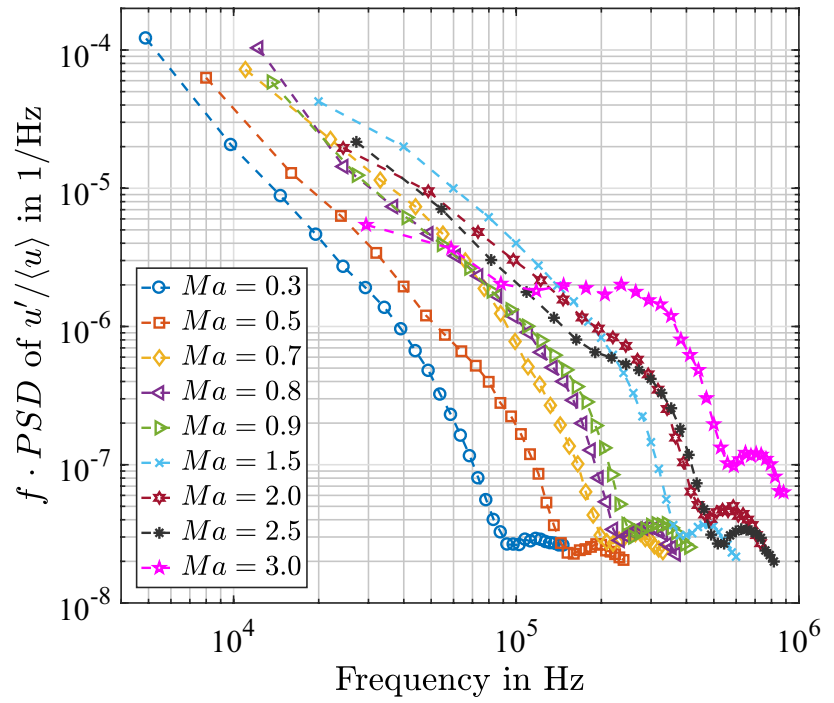

Fig. 13 Power spectral density of the normalized velocity fluctuations for different Mach numbers. The total pressure is 1.5 bar for $M a<1$ and for the supersonic cases the lowest pressure was analyzed (compare Fig. 2)

\section{Summary and conclusions}

The turbulence level of the trisonic wind tunnel Munich was determined to be $\mathrm{Tu}=1.9 \%$ for a Mach number of $M a=0.3$ and it decreases with increasing Mach number to $\mathrm{Tu}=0.45 \%$ for $M a=3.0$.

From the sensitivity studies in Sect. 3 it can be concluded that, PIV and PTV are well suited to reliably estimate wind tunnel turbulence levels even in the case of compressible flows with significant temperature fluctuations under the following conditions:

- The PIV interrogation window size $D_{\text {I }}$ projected on the measurement plane must be small compared to the dominant turbulent structures. Otherwise the application of PTV is recommended.

- The particle image displacement $\Delta x$ must be large on the image plane to reduce the relative uncertainty.

- In contrast, the particle displacement on the measurement plane must be small to avoid bias errors due to velocity gradients. Which means the magnification must be sufficiently large.

- A variation of the particle image displacement $\Delta x$ allows to determine the measurement uncertainty.

- A variation of the interrogation window size $D_{\mathrm{I}}$ allows bias errors due to spatial filtering to be detected.

If these conditions are not fulfilled, turbulence intensities can easily be estimated incorrectly. On the one hand, the measurement uncertainty increases the estimated fluctuations and on the other hand, the interrogation window size acts as a spatial low-pass filter and decreases the estimated values. Thus, for the case of an unknown turbulent flow and an unknown measurement uncertainty, there is no way of even knowing whether the actual value is underestimated or overestimated, if only one combination of $\Delta t$ and one $D_{\mathrm{I}}$ is used.

To avoid time-consuming and expensive variations of $\Delta t$ the turbulent structures must be well resolved, such that a large displacement on the image plane as well as a large interrogation window can be used. Thus, for accurate PIV/ PTV measurements it is recommended to use camera sensors with large number of pixels or multiple camera approaches which allow for high resolution and large dynamic spatial range at the same time. Finally, a variation of the interrogation window size is always recommended to achieve confidence on the estimated results.

Acknowledgements This work is supported by the Priority Programme SPP 1881 Turbulent Superstructures funded by the German Research Foundation (Deutsche Forschungsgemeinschaft-DFG) under the project number KA1808/21-1.

Open Access This article is distributed under the terms of the Creative Commons Attribution 4.0 International License (http://creativeco mmons.org/licenses/by/4.0/), which permits unrestricted use, distribution, and reproduction in any medium, provided you give appropriate credit to the original author(s) and the source, provide a link to the Creative Commons license, and indicate if changes were made.

\section{References}

Adrian RJ (1997) Dynamic ranges of velocity and spatial resolution of particle image velocimetry. Meas Sci Tech 8:1393. https://doi. org/10.1088/0957-0233/8/12/003

Alfredsson PH, Johansson AV, Haritonidis JH, Eckelmann H (1988) The fluctuating wall-shear stress and the velocity field in the viscous sublayer. Phys Fluids 31(5):1026-1033. https://doi. org/10.1063/1.866783

Beresh SJ, Henfling J, Spillers R (2017) Postage-Stamp PIV: Small Velocity Fields at $400 \mathrm{kHz}$ for Turbulence Spectra Measurements. 55th AIAA Aerosp Sci Meeting p:0024

Bolgar I, Scharnowski S, Kähler CJ (2018) The effect of the mach number on a turbulent backward-facing step flow. Flow Turbul Combust. https://doi.org/10.1007/s10494-018-9921-7

Charonko JJ, Vlachos PP (2013) Estimation of uncertainty bounds for individual particle image velocimetry measurements from crosscorrelation peak ratio. Meas Sci Tech 24(6):065,301. https://doi. org/10.1088/0957-0233/24/6/065301

Christensen K (2004) The influence of peak-locking errors on turbulence statistics computed from PIV ensembles. Exp Fluids 36:484-497. https://doi.org/10.1007/s00348-003-0754-2

Christensen K, Scarano F (2015) Uncertainty quantification in particle image velocimetry. Meas Sci Tech 26(7):070,201. https://doi. org/10.1088/0957-0233/26/7/070201

Cierpka C, Lütke B, Kähler CJ (2013) Higher order multi-frame particle tracking velocimetry. Exp Fluids 54(5):1533. https://doi. org/10.1007/s00348-013-1533-3 
Fuchs T, Hain R, Kähler CJ (2017) Non-iterative double-frame 2D/3D particle tracking velocimetry. Exp Fluids 58(9):119. https://doi. org/10.1007/s00348-017-2404-0

George WK, Lumley JL (1973) The laser-Doppler velocimeter and its application to the measurement of turbulence. J Fluid Mech 60(2):321-362. https://doi.org/10.1017/S0022112073000194

Hain R, Kähler CJ (2007) Fundamentals of multiframe particle image velocimetry (PIV). Exp Fluids 42:575-587. https://doi. org/10.1007/s00348-007-0266-6

Hultmark M, Vallikivi M, Bailey S, Smits A (2013) Logarithmic scaling of turbulence in smooth-and rough-wall pipe flow. J Fluid Mech 728:376-395. https://doi.org/10.1017/jfm.2013.255

Hutchins N, Nickels TB, Marusic I, Chong M (2009) Hot-wire spatial resolution issues in wall-bounded turbulence. J Fluid Mech 635:103-136. https://doi.org/10.1017/S0022112009007721

Kähler CJ (1997) Ortsaufgelöste Geschwindigkeitsmessungen in einer turbulenten Grenzschicht. Diploma thesis, Deutsches Zentrum für Luft- und Raumfahrt e.V. Köln, No. DLR-FB:97-32 (ISSN 1434-8454)

Kähler CJ, Sammler B, Kompenhans J (2002) Generation and control of particle size distributions for optical velocity measurement techniques in fluid mechanics. Exp Fluids 33:736-742. https:// doi.org/10.1007/s00348-002-0492-x

Kähler CJ, Scholz U, Ortmanns J (2006) Wall-shear-stress and nearwall turbulence measurements up to single pixel resolution by means of long-distance micro-PIV. Exp Fluids 41:327-341. https ://doi.org/10.1007/s00348-006-0167-0

Kähler CJ, Scharnowski S, Cierpka C (2012) On the uncertainty of digital PIV and PTV near walls. Exp Fluids 52:1641-1656. https ://doi.org/10.1007/s00348-012-1307-3

Kähler CJ, Scharnowski S, Cierpka C (2016) Highly resolved experimental results of the separated flow in a channel with streamwise periodic constrictions. J Fluid Mech 796:257-284

Kislaya A, Sciacchitano A (2018) Peak-locking error reduction by birefringent optical diffusers. Measure Sci Technol 29(2):025,202. https://doi.org/10.1088/1361-6501/aa97f7

Michaelis D, Neal DR, Wieneke B (2016) Peak-locking reduction for particle image velocimetry. Measure Sci Technol 27(10):104005. https://doi.org/10.1088/0957-0233/27/10/104005

Neal DR, Sciacchitano A, Smith BL, Scarano F (2015) Collaborative framework for PIV uncertainty quantification: the experimental database. Meas Sci Tech. https://doi.org/10.1088/09570233/26/7/074003

Nerger D, Kähler CJ, Radespiel R (2003) Zeitaufgeloeste PIV-Messungen an einem schwingenden SD7003-Profil bei $\mathrm{Re}=60,000$. In: Lasermethoden in der Strömungsmesstechnik. https://www. gala-ev.org/images/Beitraege/Beitraege\%202003/pdf/41.pdf

Ol M, McCauliffe B, Hanff E, Scholz U, Kähler CJ (2005) Comparison of laminar separation bubble measurements on a low Reynolds number airfoil in three facilities. In: 35th AIAA fluid dynamics conference and exhibit, p 5149. https://doi. org/10.2514/6.2005-5149

Overmars E, Warncke N, Poelma C, Westerweel J (2010) Bias errors in PIV: the pixel locking effect revisited. In: 15th Int Symp on applications of laser techniques to fluid mechanics, Lisbon, Portugal, 5-8 July 2010. http://ltces.dem.ist.utl.pt/lxlaser/lxlaser2010/uploa d/1787_ysdxtb_1.12.1.Full_1787.pdf

Raffel M, Willert CE, Scarano F, Kähler CJ, Wereley ST, Kompenhans J (2018) Particle image velocimetry: a practical guide. Springer, New York

Ragni D, Schrijer F, Van Oudheusden B, Scarano F (2011) Particle tracer response across shocks measured by PIV. Exp Fluids 50(1):53-64. https://doi.org/10.1007/s00348-010-0892-2

Scarano F, Moore P (2012) An advection-based model to increase the temporal resolution of PIV time series. Exp Fluids 52(4):919-933
Scharnowski S, Kähler CJ (2013) On the effect of curved streamlines on the accuracy of PIV vector fields. Exp Fluids 54:1435. https:// doi.org/10.1007/s00348-012-1435-9

Scharnowski S, Kähler CJ (2016a) Estimation and optimization of lossof-pair uncertainties based on PIV correlation functions. Exp Fluids 57:23. https://doi.org/10.1007/s00348-015-2108-2

Scharnowski S, Kähler CJ (2016b) On the loss-of-correlation due to PIV image noise. Exp Fluids 57(7):1-12. https://doi.org/10.1007/ s00348-016-2203-z

Scharnowski S, Hain R, Kähler CJ (2012) Reynolds stress estimation up to single-pixel resolution using PIV-measurements. Exp Fluids 52:985-1002. https://doi.org/10.1007/s00348-011-1184-1

Scharnowski S, Bolgar I, Kähler CJ (2017a) Characterization of turbulent structures in a transonic backward-facing step flow. Flow Turbul Combust 98(4):947-967. https://doi.org/10.1007/s1049 4-016-9792-8

Scharnowski S, Grayson K, de Silva CM, Hutchins N, Marusic I, Kähler CJ (2017b) Generalization of the PIV loss-of-correlation formula introduced by Keane and Adrian. Exp Fluids 58(10):150

Schröder A, Schanz D, Michaelis D, Cierpka C, Scharnowski S, Kähler CJ (2015) Advances of PIV and 4D-PTV Shake-the-box for turbulent flow analysis - the flow over periodic hills. Flow Turbul Comb 95(2-3):193-209. https://doi.org/10.1007/s1049 4-015-9616-2

Sciacchitano A, Wieneke B (2016) PIV uncertainty propagation. Meas Sci Tech 27(8):084006. https://doi.org/10.1088/09570233/27/8/084006

Sciacchitano A, Scarano F, Wieneke B (2012) Multi-frame pyramid correlation for time-resolved PIV. Exp Fluids 53(4):1087-1105. https://doi.org/10.1007/s00348-012-1345-x

Sciacchitano A, Wieneke B, Scarano F (2013) PIV uncertainty quantification by image matching. Measure Sci Tech 24(4):045,302. https://doi.org/10.1088/0957-0233/24/4/045302

Sciacchitano A, Neal DR, Smith BL, Warner SO, Vlachos PP, Wieneke B, Scarano F (2015) Collaborative framework for PIV uncertainty quantification: comparative assessment of methods. Measure Sci Tech 26(074):004. https://doi.org/10.1088/0957-0233/26/7/07400 4

Shirai K, Pfister T, Büttner L, Czarske J, Müller H, Becker S, Lienhart H, Durst F (2006) Highly spatially resolved velocity measurements of a turbulent channel flow by a fiber-optic heterodyne laser-Doppler velocity-profile sensor. Exp Fluids 40:473-481. https://doi.org/10.1007/s00348-005-0088-3

Taylor GI (1938) The spectrum of turbulence. Proc R Soc Lond Ser A Math Phys Sci 164:476-490. https://www.jstor.org/stable/ pdf/97077.pdf?refreqid=excelsior\%3A98015b1b6f3a04d5f3 $6 \mathrm{c} 58 \mathrm{ef} 9 \mathrm{c} 145 \mathrm{e} 5 \mathrm{~b}$

Timmins BH, Wilson BW, Smith BL, Vlachos PP (2012) A method for automatic estimation of instantaneous local uncertainty in particle image velocimetry measurements. Exp Fluids 53(4):1133-1147. https://doi.org/10.1007/s00348-012-1341-1

Welch PD (1967) The Use of Fast Fourier Transform for the Estimation of Power Spectra: A Method Based on Time Averaging Over Short, Modified Periodograms. IEEE Trans Audio Electroacoustics AU- 15:70-73. https://doi.org/10.1109/TAU.1967.1161901

Wernet M, Opalski A (2004) Development and application of a MHz frame rate digital particle image velocimetry system. In: 24th AIAA aerodynamic measurement technology and ground testing conference, p 2184. https://doi.org/10.2514/6.2004-2184

Wieneke B (2015) PIV uncertainty quantification from correlation statistics. Measure Sci Tech 26(7):074002. https://doi. org/10.1088/0957-0233/26/7/074002

Willert CE, Cuvier C, Foucaut J, Klinner J, Stanislas M, Laval J, Srinath S, Soria J, Amili O, Atkinson C, Kähler CJ, Scharnowski S, Hain R, Schröder A, Geisler R, Argocs J, Röse A (2018) Experimental evidence of near-wall reverse flow events in a zero 
pressure gradient turbulent boundary layer. Exp Thermal Fluid Sci 91:320-328. https://doi.org/10.1016/j.expthermflusci.2017.10.033

Wilson BM, Smith BL (2013) Uncertainty on PIV mean and fluctuating velocity due to bias and random errors. Measure Sci Tech 24(3):035,302. https://doi.org/10.1088/0957-0233/24/3/035302

Xue Z, Charonko JJ, Vlachos PP (2015) Particle image pattern mutual information and uncertainty estimation for particle image velocimetry. Measure Sci Tech 26(7):074,001
Publisher's Note Publisher's Note Springer Nature remains neutral with regard to jurisdictional claims in published maps and institutional affiliations. 